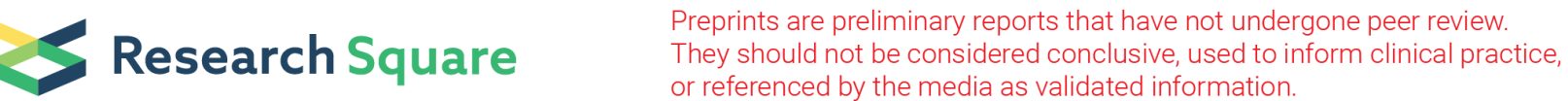

\section{Long Non-coding RNA SNHG25 Promotes the Malignancy of Endometrial Cancer by Sponging microRNA-497-5p and Thereby Increasing FASN Expression}

\author{
Yuhua He \\ Jinshan District Tinglin Hospital \\ Shuifang Xu \\ Jishan District Tinglin Hospital \\ Yi Qi \\ Jinshan District Tinglin Hospital \\ Jinfang Tian \\ Jinshan District Tinglin Hospital \\ Fengying Xu ( $\nabla$ xu.fengying@jsdtl-hospitalsh.cn ) \\ Jinshan District Tinglin Hospital https://orcid.org/0000-0001-9880-8283
}

\section{Research Article}

Keywords: SNHG25, endometrial cancer, ceRNA, miRNA sponge

Posted Date: September 1st, 2021

DOI: https://doi.org/10.21203/rs.3.rs-835767/v1

License: (c) (i) This work is licensed under a Creative Commons Attribution 4.0 International License.

Read Full License 


\section{Abstract}

\section{Background}

Small nucleolar RNA host gene 25 (SNHG25), a long-noncoding RNA, has been well studied in epithelial ovarian cancer. Yet, the specific functions of SNHG25 in endometrial cancer (EC) have not been researched. In this study, we proposed to expose the clinic significance of SNHG25 in EC, and then unravel the regulatory activity of SNHG25 on the tumor-associated phenotype of EC. More interestingly, the possible molecular events occurred when SNHG25 executives its function in EC were explored thoroughly.

\section{Methods}

We measured genes expression applying quantitative real-time polymerase chain reaction. The detailed functions of SNHG25 in EC were examined employing loss-of-function experiments. What's more, we unveiled the regulatory mechanisms among SNHG25, microRNA-497-5p and fatty acid synthase (FASN) with the application of luciferase reporter assay and RNA Immunoprecipitation.

Results

We verified a high level of SNHG25 in EC through TCGA dataset and our own cohort. Patients with a high SNHG25 level featured shorter overall survival in contrast to patients with a low SNHG25 level. SNHG25 deficient caused tumor-repressing actions in EC cells by decreasing cell proliferation, migration and invasion and promoting cell apoptosis. Furthermore, we certified the function of SNHG25 depletion in impairing tumor growth in vivo. With respect to the mechanisms, SNHG25 sequestered miR-497-5p as a competing endogenous RNA in EC and consequently positively regulated FASN expression. Striking, the decrease of miR-497-5p or increase of FASN could neutralize the modulatory actions of SNHG25 knockdown in EC cells.

\section{Conclusions}

Depleted SNHG25 hampered the oncogenicity of EC by targeting miR-497-5p/FASN axis. The newly certified SNHG25/miR-497-5p/FASN pathway may potentially have usefulness as a promising target for molecular targeted management.

\section{Background}

Endometrial cancer (EC) ranks the fourth most frequently gynecological tumor among women in the world [1]. The morbidity of EC is in a gradually ascending trend recently, and age of EC is becoming younger[2]. Every year, approximately 380,000 EC cases were happened, and caused 89,000 deaths globally[3]. At present, thanks to the substantial improvement of therapeutic techniques, patients with EC usually get favorable outcome, and their 5-year survival is about 85\%[4]. But, a large part of patients with EC are diagnosed at advanced stage at the time of symptom onset, and these therapeutic interventions 
are not effective for those patients, with a 5-year survival of less than $20 \%$ [5]. Many risk factors have been exposed to be implicated in EC pathogenesis; yet, the principle molecular events occurred downstream of this disease remain elusive[6]. Thus, an increased knowledge of EC onset and progression at the molecular level may perform essential roles in treating EC, and consequently achieve better therapeutic efficacy.

Genetic research have illustrated that non-coding RNAs account for the vast majority of the human genome[7]. Long-noncoding RNAs (IncRNA) constitute a group of non-coding transcripts whose length is over 200 nucleotides[8]. Although they are unable to be translated into protein, they exert crucial actions in dozens of human disorders[9]. Nowadays, the dysregulation of IncRNAs is certified in almost all human cancer types, and exert as cancer promoters or cancer inhibitors[10]. Notably, a considerable amount of studies have proved that accumulated IncRNAs are differentially expressed in EC, and take part in diverse malignant procedures during endometrial carcinogenesis and progression[11-13].

MicroRNAs (miRNAs) are 17-23 nt RNA transcripts which are short of protein-coding capacity[14]. They work via base pairing to the 3\-untranslated regions (3囚-UTRs) of their target genes, consequently result in translational suppression or mRNAs degradation[15]. Through affecting tumor-associated genes, miRNAs execute tumor-promoting or tumor-repressing roles during oncogenesis and cancer progression[16]. Importantly, IncRNAs are capable to competitively bind to miRNAs and thereby separate miRNA away from their targets, finally constructing the competing endogenous RNA (ceRNA) theory[17]. Therefore, investigation on IncRNAs, miRNAs and relevant working pathway may aid in the management of EC.

SNHG25 has been well studied in epithelial ovarian cancer[18]. Nevertheless, the specific functions of SNHG25 in EC have not been researched. In this study, we proposed to expose the clinic significance of SNHG25 in EC, and then unravel the regulatory activity of SNHG25 on the tumor-associated phenotype of EC. More interestingly, the possible molecular events occurred when SNHG25 executes its function in EC were explored thoroughly. All results may afford a theoretical foundation for developing novel biomarkers for treating EC.

\section{Methods}

\section{Clinical samples and cell lines}

This research was implemented under the permission of the Ethics Committee of Jinshan District Tinglin Hospital. After obtaining the signed informed consent, totally 46 pairs of EC tissues and adjacent normal tissues were gathered from our hospital. All patients had not received preoperative hormone therapy, radiotherapy or chemotherapy. Tissues were preserved in liquid nitrogen till use.

EC cell lines, HEC-1-B and AN3CA, were grown in Minimum Essential Medium (Gibco; Thermo Fisher Scientific, Inc., Waltham, MA, USA), while DMEM:F12 medium (Gibco) was used for RL95-2 and KLE cell lines. All aforementioned cells were acquired from American Type Culture Collection (ATCC; Manassas, VA, USA). Meanwhile, $10 \%$ fetal bovine serum (FBS) and $1 \%$ penicillin/streptomycin mixture (Gibco) was 
added in culture medium. Human endometrial epithelial cells were cultured in complete culture medium for human endometrial epithelial cells (both from Procell Life Science \& Technology Co., Ltd.; Wuhan, China). All cells were maintained in a humidified atmosphere of $5 \% \mathrm{CO}_{2}$ at $37^{\circ} \mathrm{C}$.

\section{Transfection assay}

Shanghai GenePharma Co., Ltd., (Shanghai, China) designed and synthesized the small interfering RNA (siRNA) targeted SNHG25 (si-SNHG25) and negative control (NC) siRNA (si-NC). FASN overexpression plasmid pcDNA3.1-FASN was prepared by GenScript Biotech Corp,(Nanjing, China). The miRNA oligonucleotides, including miR-497-5p mimic, NC mimic, miR-497-5p inhibitor (anti-miR-497-5p), and NC inhibitor (anti-NC), were also acquired from Shanghai GenePharma Co., Ltd. Transfection experiment was conducted applying Lipofectamine ${ }^{\circledR} 2000$ (Invitrogen; Thermo Fisher Scientific, Inc.).

\section{Quantitative real-time polymerase chain reaction (qRT-PCR)}

Total RNA abstraction was performed utilizing TRIzol (Invitrogen, Carlsbad, CA, USA). Total RNA was reverse transcribed into cDNA by means of PrimeScript ${ }^{\mathrm{TM}} \mathrm{RT}$ reagent Kit (Takara; Dalian, China). Next, the TB Green ${ }^{\circledR}$ Premix Ex Taq ${ }^{\text {TM }}$ II (Takara) was used for SNHG25 and FASN measurement. GAPDH acted as the reference control for SNHG25 and FASN expression. For miR-497-5p expression determination, miRNA-specific complementary DNA was synthesized via Mir-X miRNA First-Strand Synthesis Kit, after which were subjected to PCR reaction with Mir-X miRNA qRT-PCR TB Green® Kit (Takara). Small nuclear RNA U6 served as the reference gene for miR-497-5p. The $2^{-\Delta \Delta C q}$ method was applied for gene expression calculation.

\section{Subcellular fractionation experiment}

EC cells were subjected to a PARIS kit (Thermo Fisher Scientific, Inc.) for nuclear and cytoplasmic fractions separation. The RNA from nuclear and cytoplasmic fractions was extracted, and then analyzed with qRT-PCR for relative SNHG25 distribution assessment.

\section{Cell Counting Kit-8 (CCK-8) assay}

Preparation of cell suspension was executed by mixing $2 \times 10^{3}$ cells with $1 \mathrm{ml}$ complete culture medium. Each well of 96 -well plate was covered with $100 \mu \mathrm{l}$ cell suspension, followed by cultivation for different time points. After that, cells received $2 \mathrm{~h}$ incubation utilizing CCK-8 reagent (Beyotime; Shanghai, China) at $37^{\circ} \mathrm{C}$ for $2 \mathrm{~h}$. Finally, a microplate reader was used to monitor the absorbance at $450 \mathrm{~nm}$ wavelength.

\section{Transwell migration and invasion experiments}

For migration experiments, cell suspension was made by adding $5 \times 10^{5}$ cells into $1 \mathrm{ml}$ FBS-free culture medium. The upper chambers of Transwell inserts (BD Biosciences) were covered with $200 \mathrm{ul}$ suspension. Complete culture medium supplemented with $20 \%$ FBS was added into the lower chambers. After culturing for $24 \mathrm{~h}$, the non-migrated cells were cleaned by swiping the upper layer of the membrane 
with a cotton bud. Thereafter, $4 \%$ paraformaldehyde was applied for fixing the migrated cells, followed by dyeing using $0.5 \%$ crystal violet. The experiment procedures of invasion test were the same as abovementioned, with the exception that the membranes were coated with Matrigel (BD Biosciences) before cell seeding. An inverted microscope (Leica, Wetzlar, Germany) was employed to acquire cell images and count the migrated/invaded cells in five randomly-selected visuals.

\section{Flow cytometry analysis}

Annexin V-FITC Apoptosis Detection Kit (Beyotime; Shanghai, China) was used in cell apoptosis assessment. After being washed with phosphate buffer saline, transfected cells were harvested via trypsin treatment and centrifugation, after which were resuspended in $195 \mu$ l Annexin V-FITC buffer. Cells were then stained in dark with $5 \mu \mathrm{l}$ Annexin V-FITC and $10 \mu \mathrm{l}$ propidium iodide at $20^{\circ} \mathrm{C}$ for $30 \mathrm{~min}$. The apoptotic cells was analysed utilizing a flow cytometer (BD Biosciences, Franklin Lakes, NJ, USA).

\section{Tumor xenografts in nude mice}

The animal experiment was carried out with the approval of Animal Ethics Committee of Jinshan District Tinglin Hospital. Short-hairpin RNA (shRNA) targeting SNHG25 (sh-SNHG25) and NC shRNA (sh-NC) were synthesized by Shanghai GenePharma Co., Ltd. Prior to being transfected into 293T cells in parallel with psPAX2 packaging plasmid and pMD2.G envelope plasmid, the shRNAs were inserted into the pLKO.1 plasmid. When the cells were cultivated at at $37^{\circ} \mathrm{C}$ in the presence of $5 \% \mathrm{CO}_{2}$ for $48 \mathrm{~h}$, the lentiviruses stably expressing sh-SNHG25 or sh-NC were harvested and utilized to infect HEC-1-B cells. The stable transfected cells were screen out applying Puromycin.

Female BALB/c nude mice (Vital River Laboratory Animal Technology Co., Ltd; Beijing, China), 4-6 weeks old, were randomly classified into two groups, and, respectively, received injection of HEC-1-B cells with stable sh-SNHG25 or sh-NC transfection. Tumor width and length was detected once every five days, which was applied for the calculation of tumor volume. Tumor volume was calculated with the formula: volume $\left(\mathrm{mm}^{3}\right)=0.5 \times$ length $(\mathrm{mm}) \times$ width $^{2}\left(\mathrm{~mm}^{2}\right)$. All mice were euthanized by cervical dislocation at 30 days post cell injection. Subcutaneous xenografts were separated from nude mice and weighted.

\section{Bioinformatic analysis}

We predicted the downstream targets of SNHG25 utilizing starBase version 3.0 (http://starbase.sysu.edu.cn). Also, the potential targets of miR-497-5p were searched employing TargetScan (http://www.targetscan.org/), miRDB (http://mirdb.org/) and starBase version 3.0.

\section{RNA Immunoprecipitation (RIP)}

RIP was executed to certify the binding among SNHG25, miR-497-5p and FASN with the application of an EZ-Magna RIP ${ }^{\text {TM }}$ RNA-Binding Protein Immunoprecipitation Kit (EMD Millipore; Billerica, MA, USA). Cells were treated with RIP lysis buffer and centrifuged at $1,000 \times \mathrm{g}$ at $4{ }^{\circ} \mathrm{C}$ for $15 \mathrm{~min}$. The acquired cell lysate was cultivated the whole night with magnetic beads that were conjugated with anti-Argonaute 2 (Ago2) or 
IgG antibody (EMD Millipore) at $4^{\circ} \mathrm{C}$. After detachment by protease $\mathrm{K}$, the immunoprecipitated RNA was abstracted and examined by qRT-PCR.

\section{Luciferase reporter assay}

SNHG25 and FASN sequences contained predicted wild-type (wt) miR-497-5p binding site were amplified by Shanghai GenePharma Co., Ltd., and inserted downstream of the luciferase reporter plasmid pmirGLO (Promega, Madison, Wisconsin, USA). The produced luciferase reporter plasmids were entitled as SNHG25-wt and FASN-wt, respectively. In the meantime, the luciferase reporter plasmids harbouring the mutant (mut) binding site, namely SNHG25-mut and FASN-mut, were prepared employing the same experiment procedures. After seeding into 24-well plates, wt or mut reporter plasmids in combination with miR-497-5p mimic or NC mimic were cotransfected into EC cells, followed by $48 \mathrm{~h}$ cultivation at $37^{\circ}$ for two days. The activity triggered by luciferase reporter plasmids was measured with the help of a dualluciferase reporter system (Promega).

\section{Western blotting}

After isolating total protein applying RIPA buffer, a BCA Protein Assay Kit (both from Beyotime) was adopted for total protein quantification. The same amount of protein was separated by sodium dodecyl sulfate/polyacrylamide gel electrophoresis. Subsequently, the resolving proteins were transferred onto polyvinylidene difluoride membranes and then blocked with $5 \%$ fat-free milk at room temperature for $2 \mathrm{~h}$. Next, at $4^{\circ} \mathrm{C}$, the primary antibodies, anti-FASN (ab128870) and anti-GAPDH (ab181603; Abcam, Cambridge, UK), were added to incubate the membranes all night. Following the secondary antibody (ab205718; Abcam) incubation at room temperature for $1 \mathrm{~h}$, the membranes were visualized by an Enhanced Chemiluminescence Kit (Pierce; Thermo Fisher Scientific, Inc.).

\section{Statistical analysis}

All data were expressed as mean \pm standard deviation. Comparison of data between two groups was performed applying Student's t-test. One-way ANOVA followed by Tukey's test was employed to confirm the differences among multiple groups. The expression relationship was examined via Pearson's correlation analysis. The survival analysis was implemented utilizing Kaplan-Meier method, and the overall survival curves were compared via log-rank test. $\mathrm{P}<0.05$ was considered statistically significant.

\section{Results}

\section{Loss of SNHG25 performs anti-carcinogenic activities in EC cells}

Through TCGA database, we found that SNHG25 was one of top 10 overexpressed IncRNAs in uterine corpus endometrial carcinoma (Fig.1A). As compared with normal tissues, SNHG25 level was increased in uterine corpus endometrial carcinoma tissues (Fig.1B). Furthermore, a striking upregulation of SNHG25 was observed in EC tissues compared with adjacent normal tissues (Fig.1C). A high level of SHNG25 was related with a notable shorter overall survival (Fig.1D). 
We next attempted to unravel the impacts of SHNG25 in controlling malignant behaviours of EC cells. Four EC cell lines manifested a clear overexpression of SNHG25, especially in HEC-1-B and AN3CA (Fig.2A); therefore, cell lines HEC-1-B and AN3CA were applied in subsequent experiments. si-SNHG25\#1 or si-SNHG25\#2 manifested relatively highest transfection efficacy(Fig.2B), and thus we used the two siRNAs in following assays. The proliferative ability was suppressed in EC cells upon SNHG25 depletion (Fig.2C). Additionally, SNHG25 interference clearly induced EC apoptotic cell percentage (Fig.2D). Furthermore, the migration and invasion of EC cells was impeded under si-SNHG25 transfection (Figs.2E and F). On account of the above data, SNHG25 executed tumor-promoting actions in EC cells.

\section{SNHG25 is a miR-497-5p sponge in EC}

Next, we unveiled the regulatory mechanism occurred downstream of SNHG25. By means of subcellular fractionation experiment, SNHG25 was verified as a cytoplasmic IncRNA in EC cells (Fig.3A), suggesting it may be a molecular sponge or ceRNA. Through starBase, 18 miRNAs were observed to contain interacting sites for SNHG25. Levels of the 18 miRNAs in uterine corpus endometrial carcinoma were examined applying TCGA, revealing the downregulation of miR-195-5p, miR-296-3p, miR-424-5p and miR497-5p in uterine corpus endometrial carcinoma (Figs.3B and C). Accordingly, they were selected for an in-depth certification. Subsequently, we measured the expression of four candidates in SNHG25-silenced EC cells. Transfection of si-SNHG25 apparently overexpressed miR-497-5p, whereas miR-195-5p, miR-296$3 p$, and miR-424-5p levels were unchanged in response to SNHG25 deficient (Fig.3D).

Fig.3E displayed the potential binding site of miR-497-5p in SNHG25. Luciferase reporter assay was implemented, and confirmed that ectopic miR-497-5p expression impaired the activity triggered by SNHG25-wt. Yet, once the binding sequences were mutated, the repressing activity was counteracted (Fig.3F). Furthermore, RIP assay confirmed that SNHG25 and miR-497-5p were abundant by Ago2 antibody, further implying the direct interaction between them (Fig.3G). We further illustrated the decrease level of miR-497-5p in EC tissues (Fig.3H), and validated that there existed an inverse relation between miR-497-5p and SNHG25 (Fig.3I). Viewed together, SNHG25 sponges miR-497-5p in EC.

\section{SNHG25 modulates FASN expression in EC cells via decoying miR-497-5p}

The detailed functions of miR-497-5p in EC cells were clarified too. Expression of miR-497-5p was markedly increased in EC when were transfected with miR-497-5p mimic (Fig.4A). Cell proliferation was hindered following miR-497-5p overexpression (Fig.4B). Exogenous miR-497-5p led to a notable promotion of EC cell apoptosis (Fig.4C). Besides, the migratory and invasive properties were impaired by miR-497-5p mimic treatment (Figs.4D and E).

Bioinformatic prediction disclosed a potential binding interaction between miR-497-5p and FASN 3'-UTR (Fig.5A), after which were proved by luciferase reporter assay. In contrast to those observed in NC mimic group, miR-497-5p mimic suppressed the luciferase activity of FASN-wt, whereas the luciferase activity of FASN-mut was unscathed in miR-497-5p-overexpressed EC cells (Fig.5B). Furthermore, the expression of 
FASN was downregulated by miR-497-5p mimic in EC cells (Figs.5C and D). These data firmly prove FASN as a direct target of miR-497-5p in EC.

As abovementioned, SNHG25 sequesters miR-497-5p and miR-497-5p directly targets FASN in EC; therefore, we next elucidated whether SNHG25 is taken part in the modulation of miR-497-5p/FASN axis. At the beginning, the expression relationship among them was detected. Overexpressed FASN in EC tissues (Fig.5E) was negatively related with miR-497-5p (Fig.5F), but exhibited a positive relation with SNHG25 (Fig.5G). Furthermore, FASN expression was downregulated when SNHG25 was ablated (Figs.5H and I), which was recovered by anti-miR-497-5p cotransfection (Figs.5J and K). Finally, RIP assay uncovered that SNHG25, miR-497-5p and FASN were all enriched by Ago2 antibody in EC cells (Fig.5L). All in all, SNHG25 functions as a ceRNA for miR-497-5p and consequently increase FASN expression.

\section{si-SNHG25 exerts a cancer-repressing role in EC via the adjustment of miR-497-5p/FASN axis}

Eventually, we applied rescue function experiments to ascertain whether the regulatory effects of siSNHG25 in EC cells were occurred through miR-497-5p/FASN axis. The transfection efficiency of antimiR-497-5p was examined and confirmed by qRT-PCR (Fig.6A). The proliferation of SNHG25-depleted EC cells was striking declined, and their cell apoptosis was promoted; yet, the modulatory actions were both eliminated by anti-miR-497-5p cotransfection (Figs.6B and C). Additionally, lowering miR-497-5p was capable to reverse the repressing effects of si-SNHG25 on EC cell migration and invasion (Figs.6D and E). Furthermore, transfection with pcDNA3.1-FASN triggered a clear upregulation of FASN (Fig.7A).

Downregulation of SNHG25 obviously restrained cell proliferation and facilitated cell apoptosis; however, the treatment of pcDNA3.1-FASN was sufficient to abolish the effects (Figs.7B and C). Besides, the migratory and invasive abilities of si-SNHG25-transfected cells were hindered, but these changes were reversed upon FASN reintroduction (Figs.7D and E). Altogether, miR-497-5p/FASN axis served as the downstream effector of SNHG25 in EC.

\section{Interference of SNHG25 impedes tumor growth in vivo}

Tumor xenografts in nude mice were realized to test the impact of SNHG25 interference on tumor growth in vivo. In comparison with sh-NC group, tumor xenografts originated from SNHG25-silenced cells presented smaller sizes (Figs.8A and B) and lighter weight (Fig.8C). Additionally, downregulated SNHG25 (Fig.8D), overexpressed miR-497-5p (Fig.8E) and decreased FASN (Fig.8F) was proved in sh-SNHG25transfected tumor xenografts. In short, the absence of SNHG25 restrained tumor growth in vivo.

\section{Discussion}

LncRNAs as a novel research focus harvest a considerable attention from scholars in the recent decades[19-21]. The detailed roles of IncRNAs have been intensively studied in EC oncogenesis and progression[22-24]. A substantial amount of IncRNAs are differentially expressed in EC and take part in the control of numerous aggressive behaviors[25-27]. In spite of that the human genome contains over 
50,000 IncRNAs[28], their enrollment in EC are not completely explored. Herein, we proposed to examine whether SNHG25 exerts a significant role in EC and the relevant working mechanisms.

SNHG25 is a poorly understood IncRNA. SNHG25 is upregulated in epithelial ovarian cancer, displaying a significant correlation with histological grade[18]. Overexpressed SNHG25 hinders epithelial ovarian cancer cell apoptosis but increases cell proliferation, migration and invasion[18]. Yet, whether SNHG25 presents clinical value in EC and how it performs its detailed roles is still unbeknown. In this study, we verified a high level of SNHG25 in EC through TCGA dataset and our own cohort. Patients with a high SNHG25 level featured shorter overall survival in contrast to patients with a low SNHG25 level. Functionally, SNHG25 deficient caused tumor-repressing actions in EC cells by decreasing cell proliferation, migration and invasion and promoting cell apoptosis. Furthermore, we certified that the function of SNHG25 depletion in impairing tumor growth in vivo. Accordingly, SNHG25 might be a potential target for diagnosing and treating EC.

The molecular events played by IncRNAs are largely dependent upon their subcellular location[29]. The nuclear IncRNAs are capable to affect genes expression at transcriptional level through interacting with proteins[30]. Instead, the ceRNA theory is widely actualized by cytoplasmic IncRNAs, thereby controlling genes expression at post-transcriptional level[31]. In this context, they harbor miRNA-response elements, can decoy with miRNAs, and consequently abstracting miRNAs from their targets[17]. To unravel the mechanisms underlying the actions of SNHG25, we firstly applied subcellular fractionation experiment to affirm the localization of SNHG25 in EC cells. SNHG25 was turned out to be primarily distributed in EC cell cytoplasm, implying a role for SNHG25 as a ceRNA.

Utilizing bioinformatics analysis, the complementary binding sequences between SNHG25 and miR-497$5 p$ were assumed. Importantly, this prediction was further acknowledged by multiple mechanistic investigations, which manifested SNHG25 as an miR-497-5p sponge. Additionally, evidence was offered that miR-497-5p directly targeted FASN in EC. MiR-497-5p downregulated FASN expression through direct interaction with its 3'-UTR. Furthermore, FASN was under the positive control of SNHG25 in EC cells, which was happened via sequestering miR-497-5p. Notably, as confirmed by RIP, the aforementioned three RNAs were all substantially enriched by Ago2. That is to say, our study gives clear evidence to propose a new ceRNA pathway in EC, comprising SNHG25, miR-497-5p and FASN.

A low miR-497-5p level was observed in melanoma[32], non-small-cell lung cancer[33], ovarian cancer[34] and cervical cancer, exerting tumor-repressing activities. MiR-497-5p presents a notable correlation with the tumorigenesis of EC[35]; but, the detailed functions of miR-497-5p in EC remain elusive. In this study, we found that miR-497-5p was weakly expressed in EC and realized anticarcinogenic actions during EC oncogenesis and progression. FASN, a central lipogenic enzyme, was proved as a downstream target of miR-497-5p in EC. Overexpressed FASN in EC displayed a clear relationship with multiple aggressive clinicopathological parameters [36]. FASN served as an important mediator of the oncogenicity of EC, and controlled several biological characteristics [37-39]. Striking, the final rescue experiments illuminated that decrease of miR-497-5p or increase of FASN could neutralize the 
modulatory actions of SNHG25 knockdown in EC cells. In short, miR-497-5p/FASN axis operated as the downstream effector through which SNHG25 played cancer-promoting roles in EC.

In summary, our research uncovered an increased level of SNHG25 in EC. Depleted SNHG25 hampered the oncogenicity of EC. With respect to the mechanisms, SNHG25 sequestered miR-497-5p as a ceRNA in EC and consequently positively modulated FASN expression. Therefore, the newly certified SNHG25/miR497-5p/FASN pathway may potentially have usefulness as a promising target for molecular targeted management.

\section{Abbreviations}

EC Endometrial cancer

FBS Fetal bovine serum

NC Negative control

IncRNA Long non-coding RNA

miRNA MicroRNA

SNHG25 Small nucleolar RNA host gene 25

FASN Fatty acid synthase

\section{Declarations}

\section{Ethics approval and consent to participate}

This research was implemented under the permission of the Ethics Committee of Jinshan District Tinglin Hospital. The animal experiment was carried out with the approval of Animal Ethics Committee of Jinshan District Tinglin Hospital.

\section{Consent for publication}

Not applicable.

\section{Availability of data and materials}

The analyzed data sets generated during the study are available from the corresponding author on reasonable request.

\section{Competing interests}

The authors declare that they have no competing interests. 


\section{Funding}

This study was supported by the fourth talent training plan of Jinshan District Health System in Shanghai (JSYQ201914).

\section{Authors' contributions}

Yuhua He and Fengying Xu designed the current study. Yuhua He, Shuifang Xu, Yi Qi, Jinfang Tian, and Fengying Xu performed all the experiments. Fengying Xu analysed all the data. Yuhua He drafted the manuscript. All authors approved the final draft.

\section{References}

1. Winterhoff B, Thomaier L, Mullany S, Powell MA: Molecular characterization of endometrial cancer and therapeutic implications. Current opinion in obstetrics \& gynecology 2020, 32(1):76-83.

2. Morice P, Leary A, Creutzberg C, Abu-Rustum N, Darai E: Endometrial cancer. Lancet 2016, 387(10023):1094-1108.

3. Erratum: Global cancer statistics 2018: GLOBOCAN estimates of incidence and mortality worldwide for 36 cancers in 185 countries. CA: a cancer journal for clinicians 2020, 70(4):313.

4. Legge F, Restaino S, Leone L, Carone V, Ronsini C, Di Fiore GLM, Pasciuto T, Pelligra S, Ciccarone F, Scambia $G$ et al: Clinical outcome of recurrent endometrial cancer: analysis of post-relapse survival by pattern of recurrence and secondary treatment. International journal of gynecological cancer : official journal of the International Gynecological Cancer Society 2020, 30(2):193-200.

5. Gupta D: Clinical Behavior and Treatment of Endometrial Cancer. Advances in experimental medicine and biology 2017, 943:47-74.

6. Kyo S, Nakayama K: Endometrial Cancer as a Metabolic Disease with Dysregulated PI3K Signaling: Shedding Light on Novel Therapeutic Strategies. International journal of molecular sciences 2020, 21(17).

7. Stein LD: Human genome: end of the beginning. Nature 2004, 431(7011):915-916.

8. Spizzo R, Almeida MI, Colombatti A, Calin GA: Long non-coding RNAs and cancer: a new frontier of translational research? Oncogene 2012, 31(43):4577-4587.

9. Chen J, Liu Y, Min J, Wang H, Li F, Xu C, Gong A, Xu M: Alternative splicing of IncRNAs in human diseases. American journal of cancer research 2021, 11(3):624-639.

10. Lopez-Jimenez E, Andres-Leon E: The Implications of ncRNAs in the Development of Human Diseases. Non-coding RNA 2021, 7(1). 
11. Liu H, Wan J, Chu J: Long non-coding RNAs and endometrial cancer. Biomedicine \& pharmacotherapy = Biomedecine \& pharmacotherapie 2019, 119:109396.

12. Li BL, Wan XP: The role of IncRNAs in the development of endometrial carcinoma. Oncology letters 2018, 16(3):3424-3429.

13. Takenaka K, Chen BJ, Modesitt SC, Byrne FL, Hoehn KL, Janitz M: The emerging role of long noncoding RNAs in endometrial cancer. Cancer genetics 2016, 209(10):445-455.

14. Hammond SM: An overview of microRNAs. Advanced drug delivery reviews 2015, 87:3-14.

15. He L, Hannon GJ: MicroRNAs: small RNAs with a big role in gene regulation. Nature reviews Genetics 2004, 5(7):522-531.

16. Rupaimoole R, Slack FJ: MicroRNA therapeutics: towards a new era for the management of cancer and other diseases. Nature reviews Drug discovery 2017, 16(3):203-222.

17. Salmena L, Poliseno L, Tay Y, Kats L, Pandolfi PP: A ceRNA hypothesis: the Rosetta Stone of a hidden RNA language? Cell 2011, 146(3):353-358.

18. Liu Y, Xu B, Liu M, Qiao H, Zhang S, Qiu J, Ying X: Long non-coding RNA SNHG25 promotes epithelial ovarian cancer progression by up-regulating COMP. Journal of Cancer 2021, 12(6):1660-1668.

19. Liu R, Wang X, Shen Y, He A: Long non-coding RNA-based glycolysis-targeted cancer therapy: feasibility, progression and limitations. Molecular biology reports 2021, 48(3):2713-2727.

20. Zhao H, De Souza C, Kumar VE, Nambiar R, Hao D, Zhu X, Luo Y, Liu S, Zhang L, Zhu J: Long non-coding RNA signatures as predictors of prognosis in thyroid cancer: a narrative review. Annals of translational medicine 2021, 9(4):359.

21. Liao Z, Nie H, Wang Y, Luo J, Zhou J, Ou C: The Emerging Landscape of Long Non-Coding RNAs in Colorectal Cancer Metastasis. Frontiers in oncology 2021, 11:641343.

22. Zhao M, Qiu Y, Yang B, Sun L, Hei K, Du X, Li Y: Long non-coding RNAs involved in gynecological cancer. International journal of gynecological cancer : official journal of the International Gynecological Cancer Society 2014, 24(7):1140-1145.

23. Yang L, Zhang J, Jiang A, Liu Q, Li C, Yang C, Xiu J: Expression profile of long non-coding RNAs is altered in endometrial cancer. International journal of clinical and experimental medicine 2015 , 8(4):5010-5021.

24. Chen BJ, Byrne FL, Takenaka K, Modesitt SC, Olzomer EM, Mills JD, Farrell R, Hoehn KL, Janitz M: Transcriptome landscape of long intergenic non-coding RNAs in endometrial cancer. Gynecologic oncology 2017, 147(3):654-662. 
25. Jian F, Che X, Zhang J, Liu C, Liu G, Tang Y, Feng W: The long-noncoding RNA SOCS2-AS1 suppresses endometrial cancer progression by regulating AURKA degradation. Cell death \& disease 2021, 12(4):351.

26. Wang L, Huang Q, Lin Q, Chen L, Shi Q: Knockdown of long non-coding RNA small nucleolar RNA host gene 9 or hexokinase 2 both suppress endometrial cancer cell proliferation and glycolysis. The journal of obstetrics and gynaecology research 2021.

27. Xin W, Gao X, Zhao P, Wang T, Ding X, Wu Q, Hua K: Long non-coding RNA RP11-379k17.4 derived microRNA-200c-3p modulates human endometrial cancer by targeting Noxa. Journal of Cancer 2021, 12(8):2268-2274.

28. Xu J, Bai J, Zhang X, Lv Y, Gong Y, Liu L, Zhao H, Yu F, Ping Y, Zhang G et al: A comprehensive overview of IncRNA annotation resources. Briefings in bioinformatics 2017, 18(2):236-249.

29. Gawronski AR, Uhl M, Zhang Y, Lin YY, Niknafs YS, Ramnarine VR, Malik R, Feng F, Chinnaiyan AM, Collins CC et al: MechRNA: prediction of IncRNA mechanisms from RNA-RNA and RNA-protein interactions. Bioinformatics 2018, 34(18):3101-3110.

30. Gao N, Li Y, Li J, Gao Z, Yang Z, Li Y, Liu H, Fan T: Long Non-Coding RNAs: The Regulatory Mechanisms, Research Strategies, and Future Directions in Cancers. Frontiers in oncology 2020, 10:598817.

31. Qi X, Zhang DH, Wu N, Xiao JH, Wang X, Ma W: ceRNA in cancer: possible functions and clinical implications. Journal of medical genetics 2015, 52(10):710-718.

32. Chai L, Kang XJ, Sun ZZ, Zeng MF, Yu SR, Ding Y, Liang JQ, Li TT, Zhao J: MiR-497-5p, miR-195$5 p$ and miR-455-3p function as tumor suppressors by targeting hTERT in melanoma A375 cells. Cancer management and research 2018, 10:989-1003.

33. Li G, Wang K, Wang J, Qin S, Sun X, Ren H: miR-497-5p inhibits tumor cell growth and invasion by targeting SOX5 in non-small-cell lung cancer. Journal of cellular biochemistry 2019, 120(6):1058710595.

34. Liu C, Bordeaux A, Hettich S, Han S: MicroRNA-497-5p Functions as a Modulator of Apoptosis by Regulating Metadherin in Ovarian Cancer. Cell transplantation 2020, 29:963689719897061.

35. Fridrichova I, Kalinkova L, Karhanek M, Smolkova B, Machalekova K, Wachsmannova L, Nikolaieva N, Kajo K: miR-497-5p Decreased Expression Associated with High-Risk Endometrial Cancer. International journal of molecular sciences 2020, 22(1).

36. Tsuji T, Yoshinaga M, Togami S, Douchi T, Nagata Y: Fatty acid synthase expression and clinicopathological findings in endometrial cancer. Acta obstetricia et gynecologica Scandinavica 2004, 83(6):586-590. 
37. Sebastiani V, Visca P, Botti C, Santeusanio G, Galati GM, Piccini V, Capezzone de Joannon B, Di Tondo U, Alo PL: Fatty acid synthase is a marker of increased risk of recurrence in endometrial carcinoma. Gynecologic oncology 2004, 92(1):101-105.

38. Anagnostou E, Miliaras D, Meditskou S, Grimbizis G: Immunohistochemical investigation of metabolic markers fatty acid synthase (FASN) and glucose transporter 1 (GLUT1) in normal endometrium, endometrial hyperplasia, and endometrial malignancy. Hippokratia 2017, 21(4):169-174.

39. Wysham WZ, Roque DR, Han J, Zhang L, Guo H, Gehrig PA, Zhou C, Bae-Jump VL: Effects of Fatty Acid Synthase Inhibition by Orlistat on Proliferation of Endometrial Cancer Cell Lines. Targeted oncology 2016, 11(6):763-769.

\section{Figures}

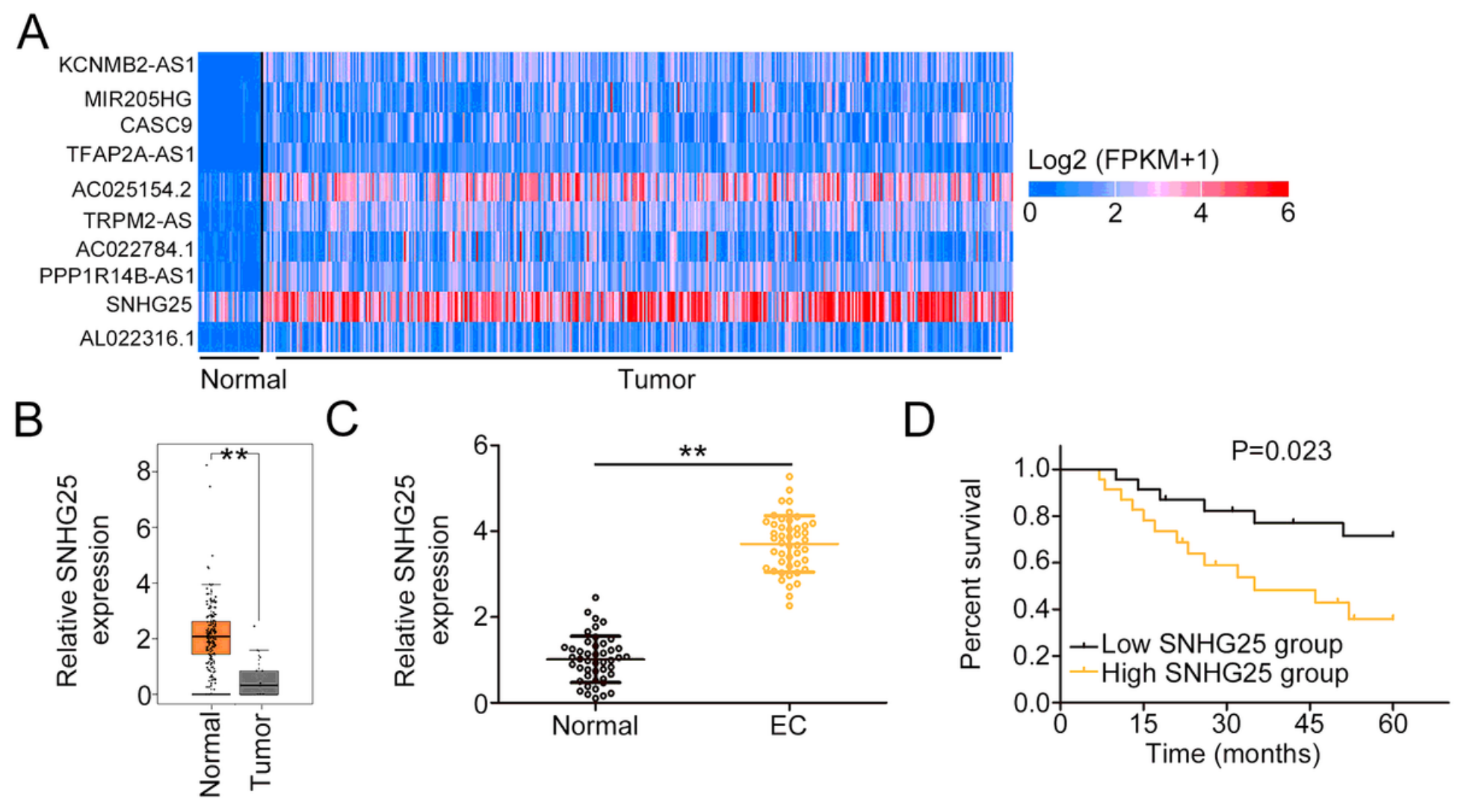

\section{Figure 1}

Increased SNHG25 level was affirmed in EC. (A) The top ten overexpressed IncRNAs in uterine corpus endometrial carcinoma. (B) Expression of SNHG25 in uterine corpus endometrial carcinoma was examined utilizing TCGA database. (C) qRT-PCR was applied for the determination of SNHG25 expression in EC tissues. (D) The relationship between SNHG25 level and overall survival in patients with EC was examined with the application of Kaplan-Meier curve. ${ }^{\star *} \mathrm{P}<0.01$. 
A

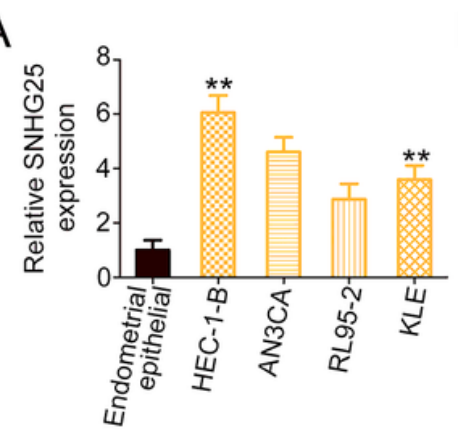

$\mathrm{D}$

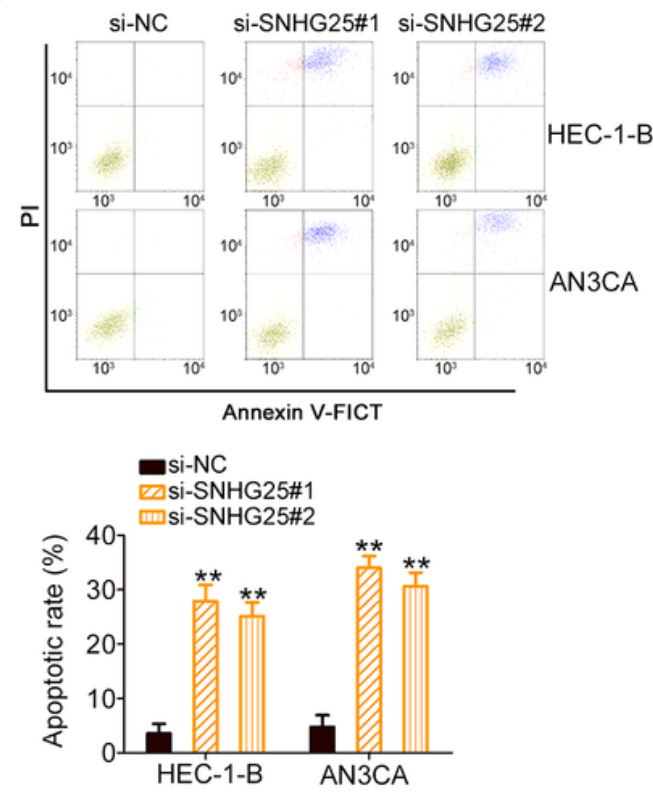

B

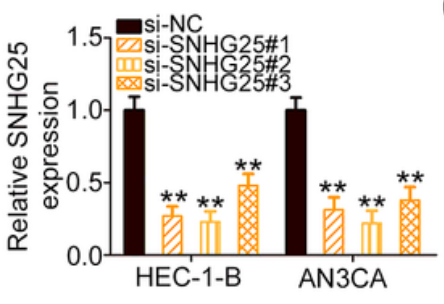

$E$
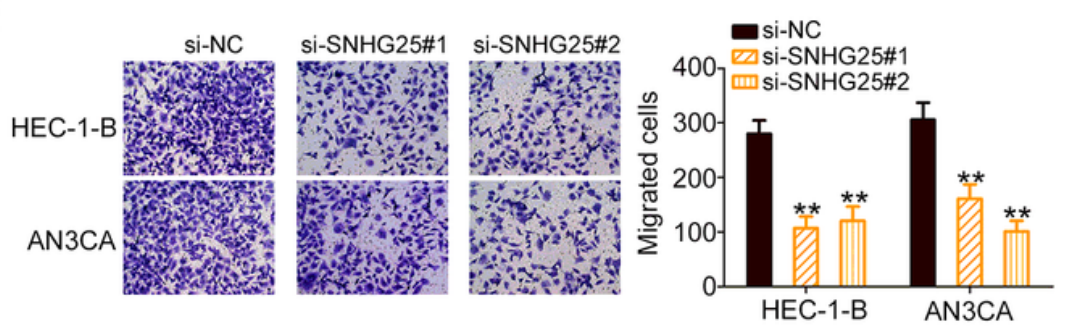

$\mathrm{F}$

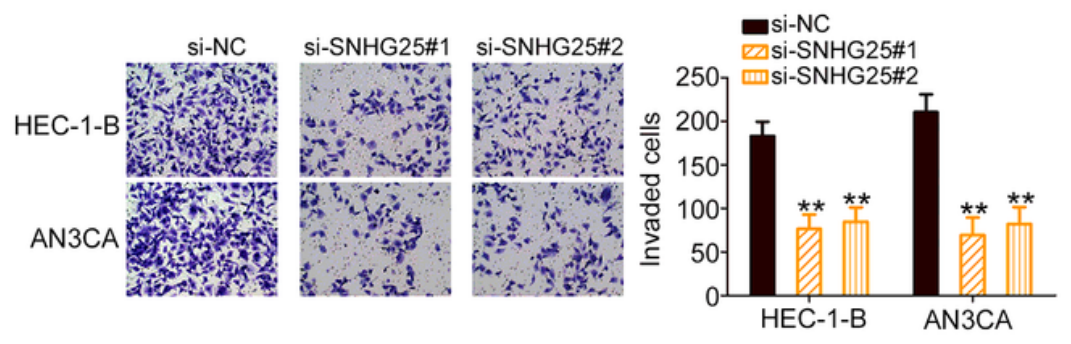

Figure 2

SNHG25 interference hampered the biological characteristics of EC cells. (A) SNHG25 expression in EC cell lines. (B) SNHG25 level in HEC-1-B and AN3CA cells overexpressing si-SNHG25 was detected via qRTPCR. (C) CCK-8 assay was implemented to evaluate the proliferation of EC cells expressing si-SNHG25. (D) The proportion of apoptotic cells were quantified in EC cells when SNHG25 was silenced. (E, F) The migratory and invasive properties of SNHG25-depleted EC cells were addressed utilizing transwell migration and invasion experiment. ${ }^{*} \mathrm{P}<0.01$. 
A

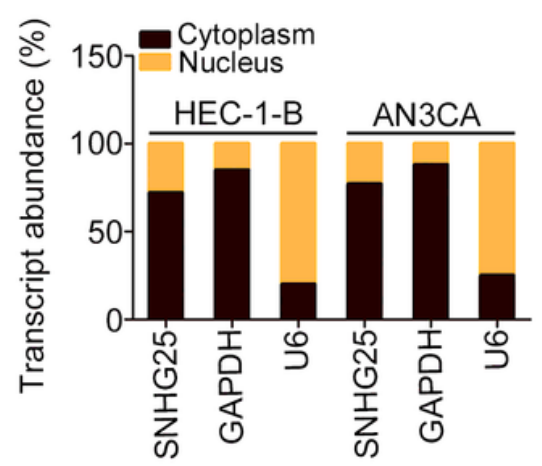

C

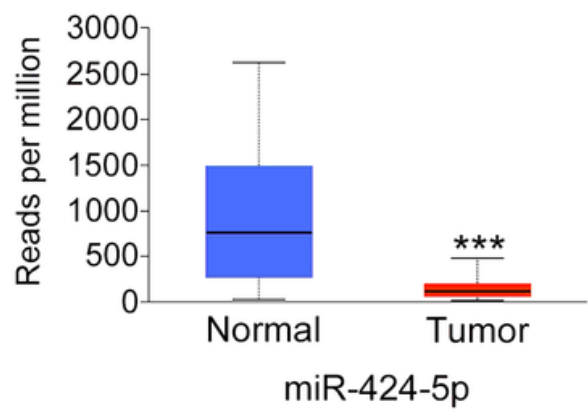

$E$ $\begin{array}{lll}\text { SNHG25-wt } & 5^{\prime} \text {...GGAUGUCAUCGUCCUUGCUGCUU... } \\ \text { miR-497-5p } & 3^{\prime} \quad \text { UGUUUGGUGUCACACGACGAC } & 5\end{array}$ SNHG25-mut 5' ...GGAUGUCAUCGUCCUACGACGAU... 3'
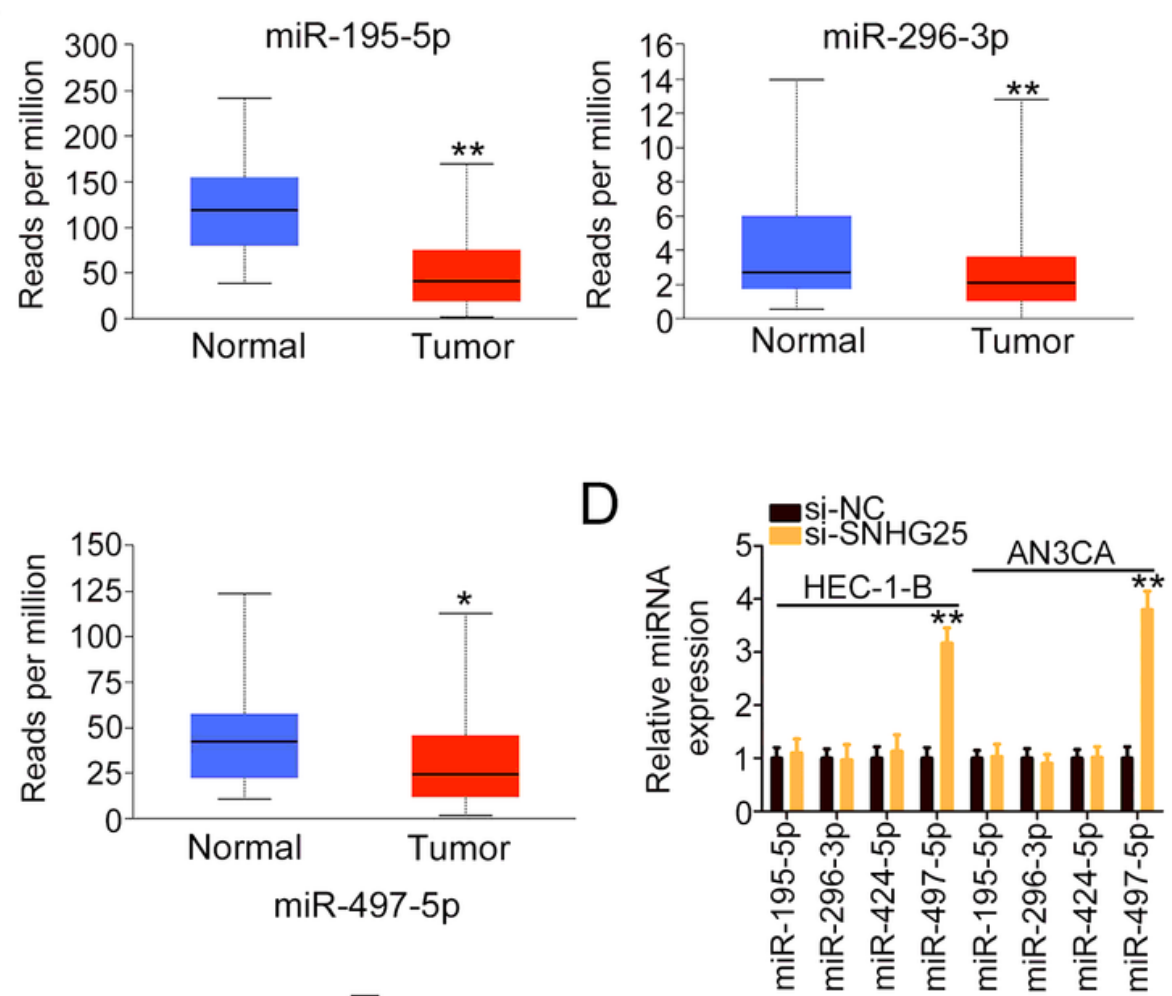

$\mathrm{F}$

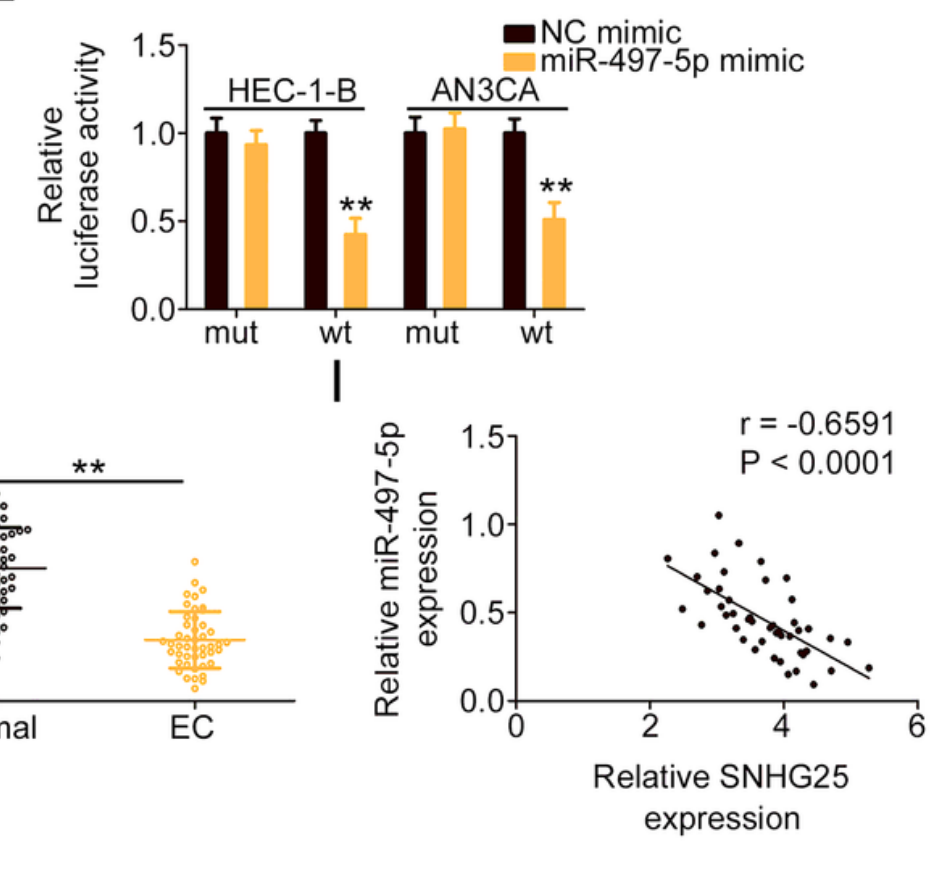

Figure 3

SNHG25 directly sponged miR-497-5p in EC. (A) The subcellular location of SNHG25 in EC cells. (B, C) Expression levels of miR-195-5p, miR-296-3p, miR-424-5p and miR-497-5p in uterine corpus endometrial carcinoma were examined utilizing TCGA database. (D) Expression of miR-195-5p, miR-296-3p, miR-424$5 p$ and miR-497-5p was tested in SNHG25-silenced EC cells. (E) The interacting sequences between SNHG25 and miR-497-5p predicted by bioinformatic analysis. (F) Luciferase activity was measured in EC cells which were transfected with SNHG25-wt or SNHG25-mut in parallel with miR-497-5p/NC mimic. (G) 
RIP assay was applied to prove the interaction between SNHG25 and miR-497-5p. (H) qRT-PCR was used in the measurement of miR-497-5p expression in EC tissues. (I) Expression correlation between miR-497$5 p$ and SNHG25 in EC tissues. **P $<0.01$.
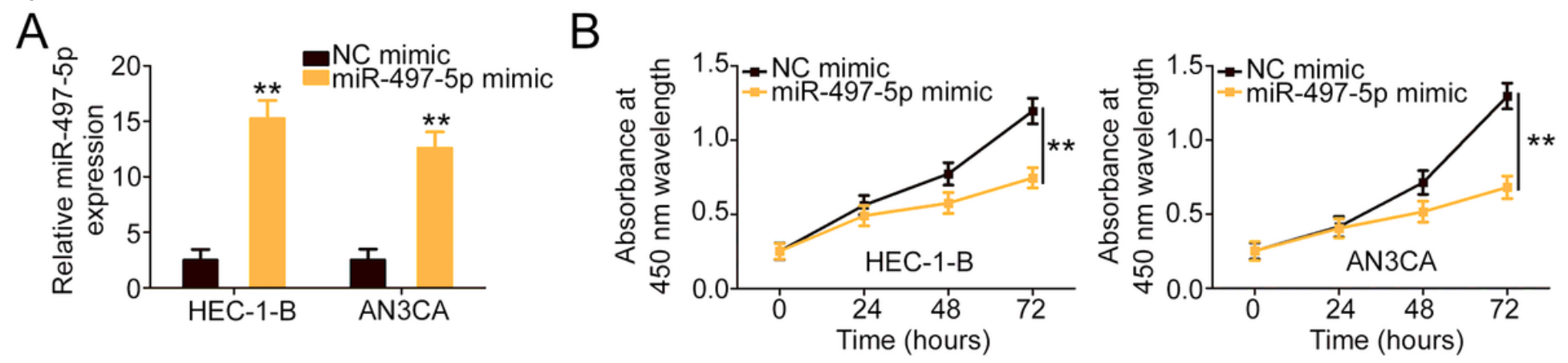

C
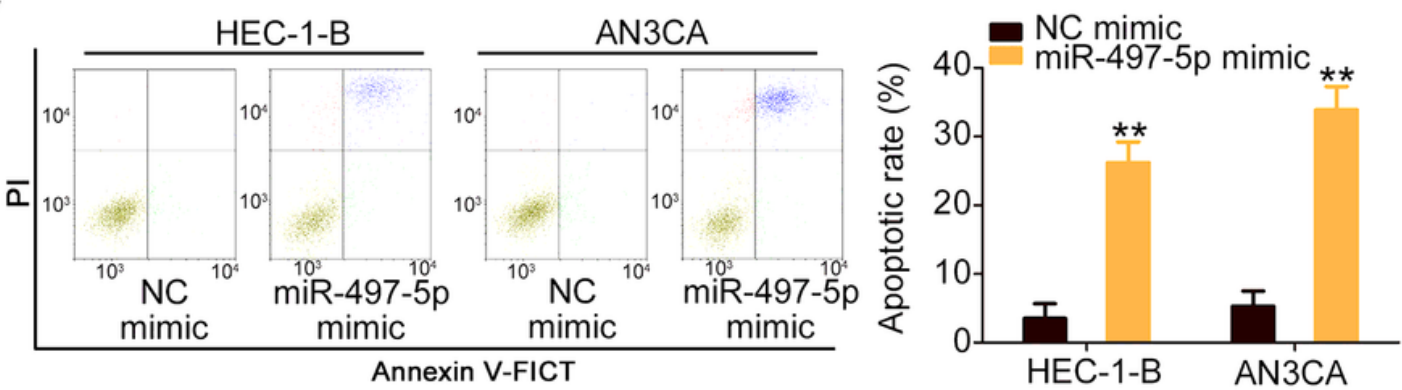

D
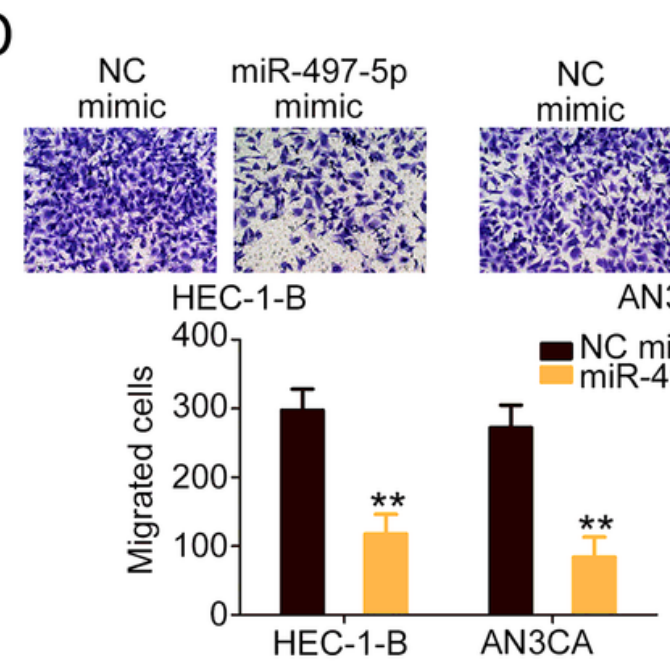

$E$

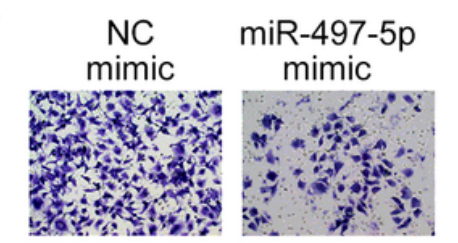

HEC-1-B

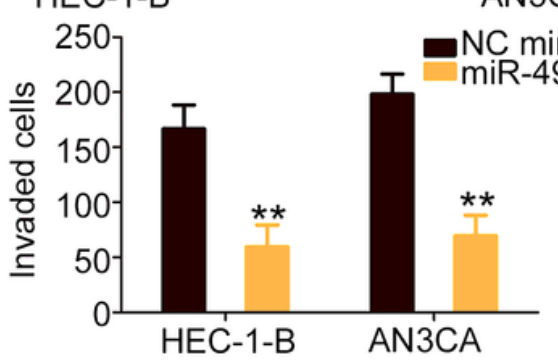

Figure 4

MiR-497-5p was certified as a tumor-inhibiting miRNA in EC. (A) The efficiency of miR-497-5p mimic transfection was examined applying qRT-PCR. (B, C) The proliferation and apoptosis of miR-497-5poverexpressed EC cells. (D, E) Cell migration and invasion was assessed in EC cells when miR-497-5p level was increased. $* * \mathrm{P}<0.01$. 
A

B

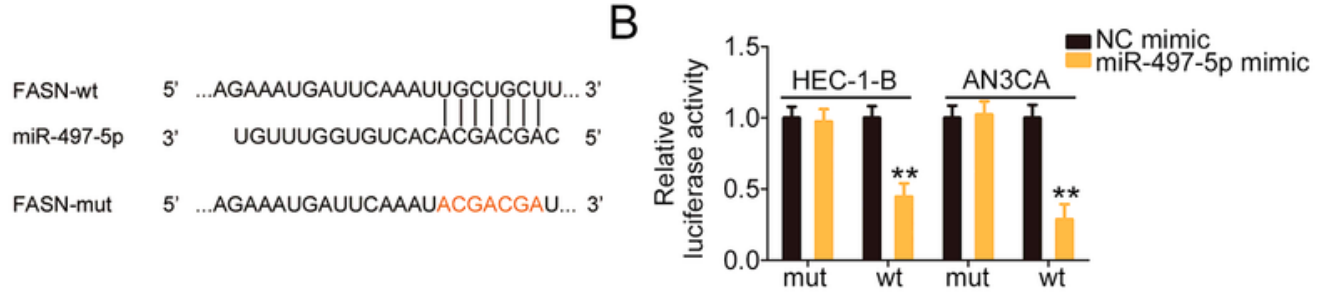

C
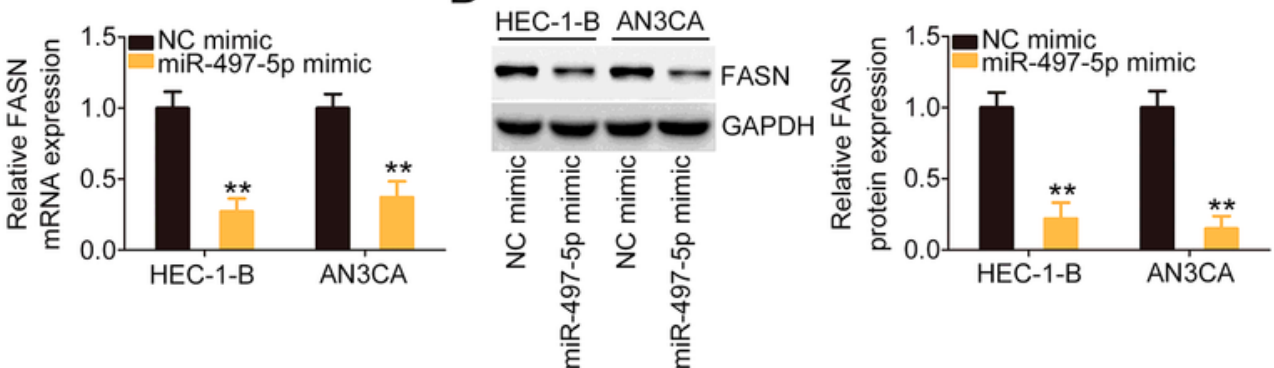

E

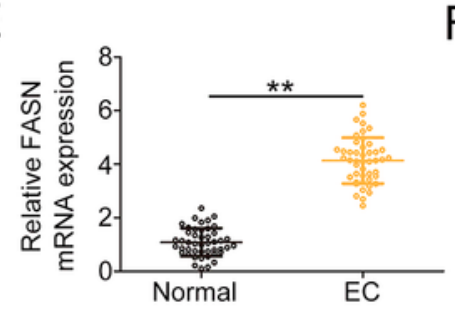

F

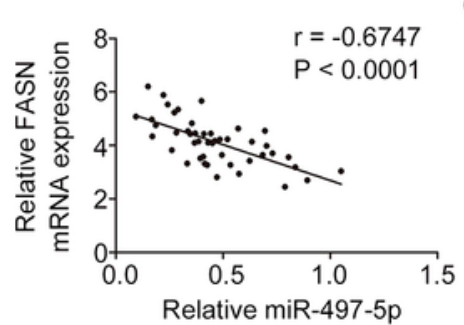

G

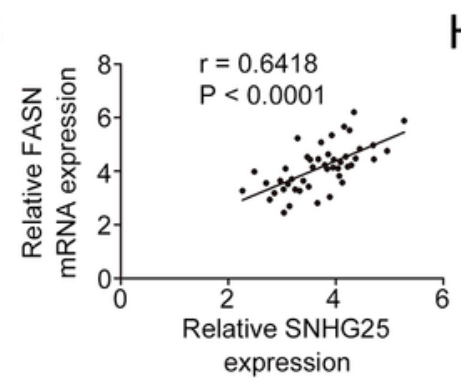

$\mathrm{H}$
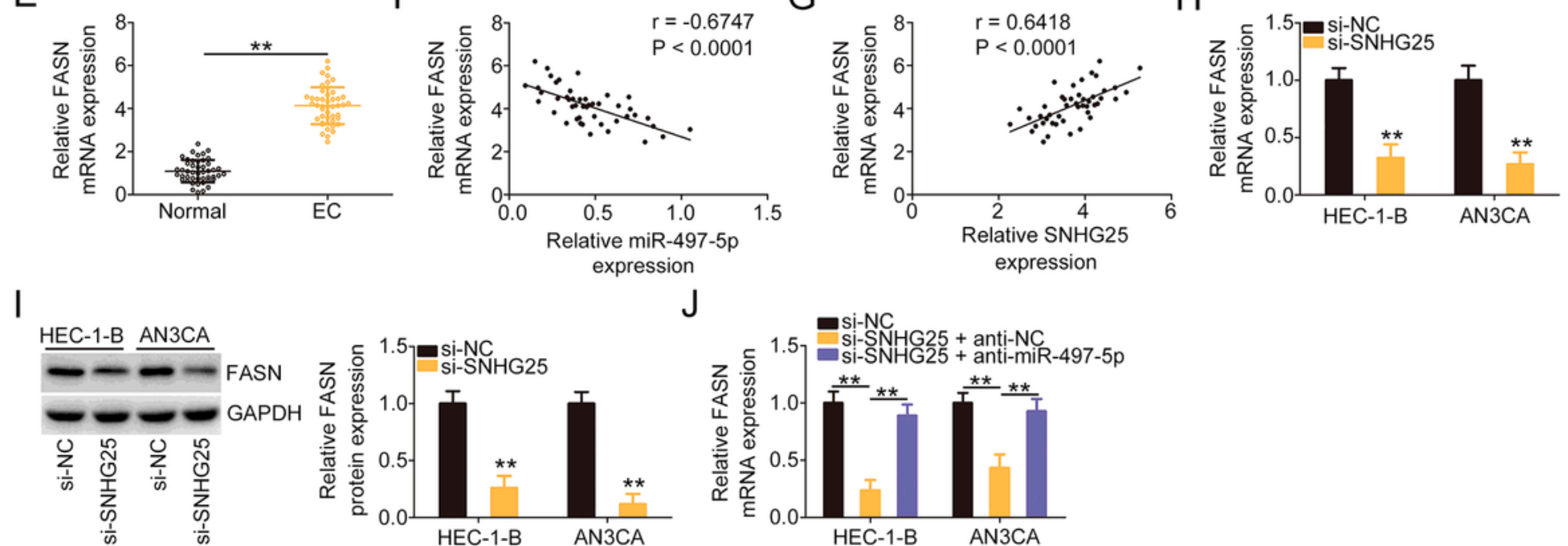

expression

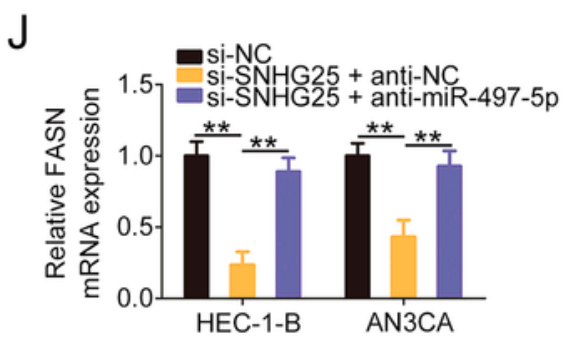

K
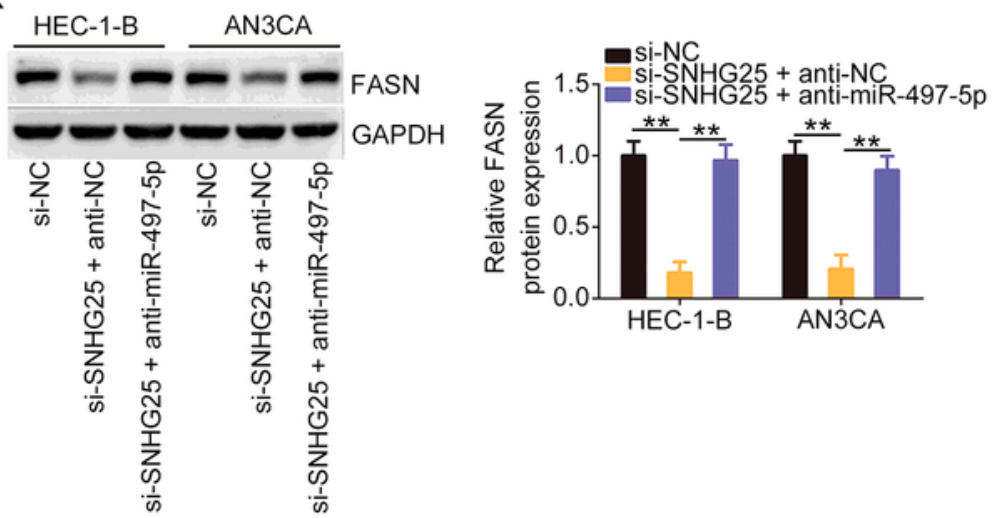

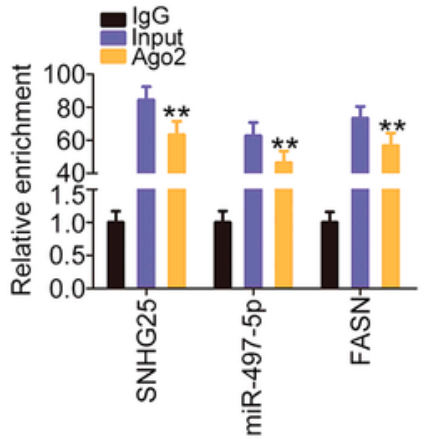

HEC-1-B

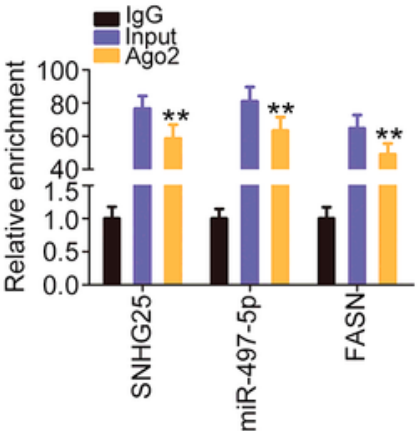

AN3CA

\section{Figure 5}

si-SNHG25 lowered FASN level through decoying miR-497-5p. (A) The interacting sequences between miR-497-5p and FASN 3'-UTR. (B) Luciferase activity was tested in EC cells which were transfected with FASN-wt or FASN-mut alongside miR-497-5p/NC mimic. (C, D) The quantification of FASN was implemented in EC cells after miR-497-5p upregulation. (E) Expression status of FASN in EC tissues. (F) Expression relation between FASN and miR-497-5p in EC tissues. (G) Expression relation between FASN 
and SNHG25 in EC tissues. $(\mathrm{H}, \mathrm{I})$ The measurement of FASN was implemented in SNHG25-deficient EC cells. (J, K) si-SNHG25 together with anti-miR-497-5p or anti-NC was transfected into EC cells, followed by the determination of FASN expression. (L) RIP assay was carried out to confirm the enrichment of SNHG25, miR-497-5p and FASN in immunoprecipitated RNA. $* * P<0.01$.

A

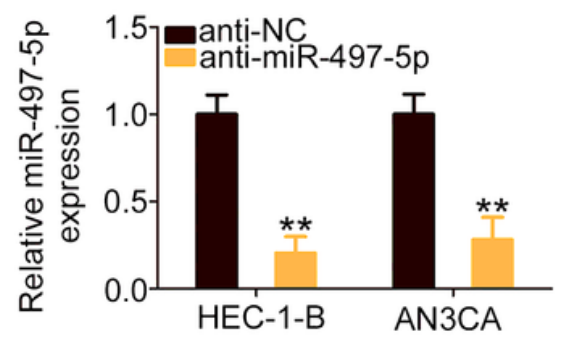

C

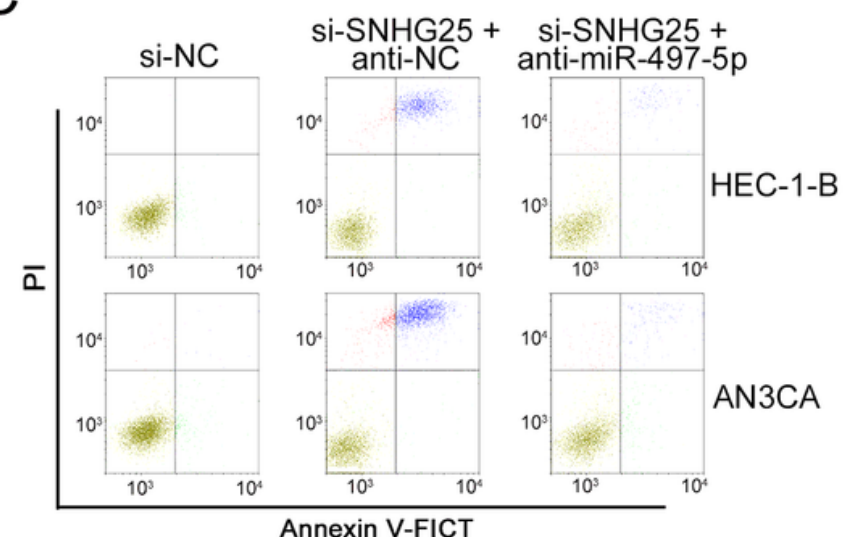

$\mathrm{D}$

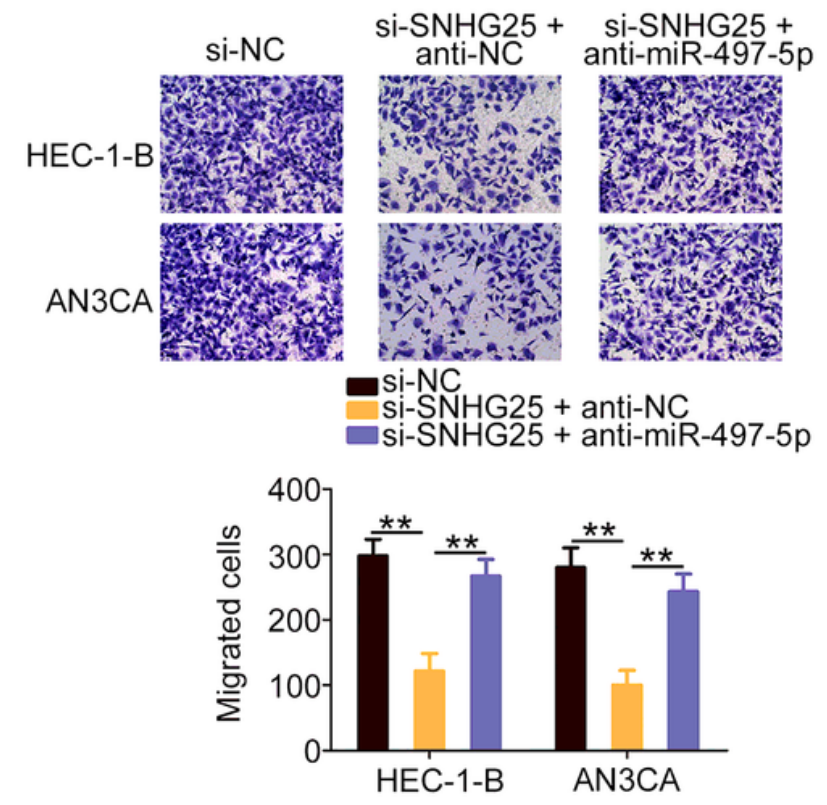

$-\mathrm{Si-NC}$

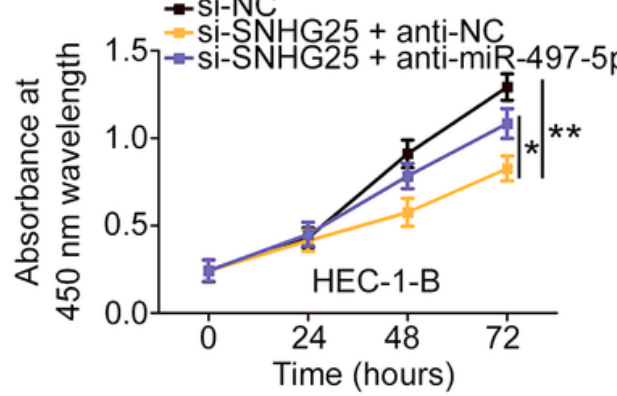

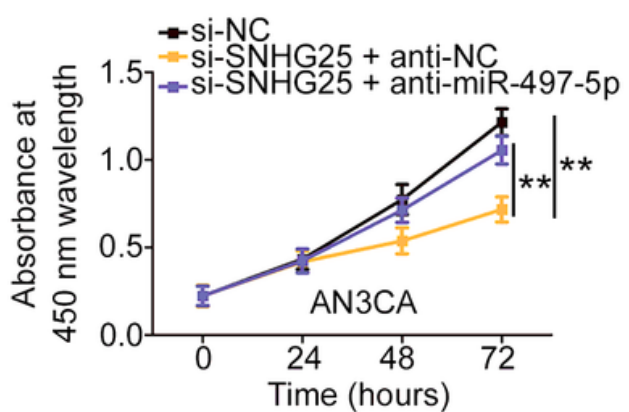

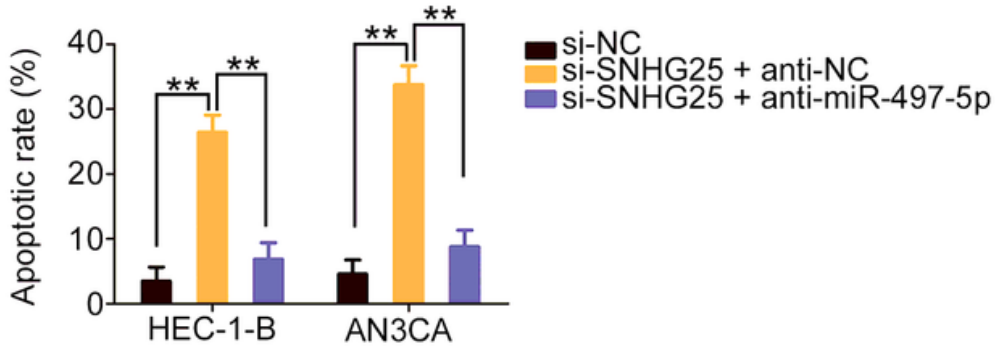

$E$
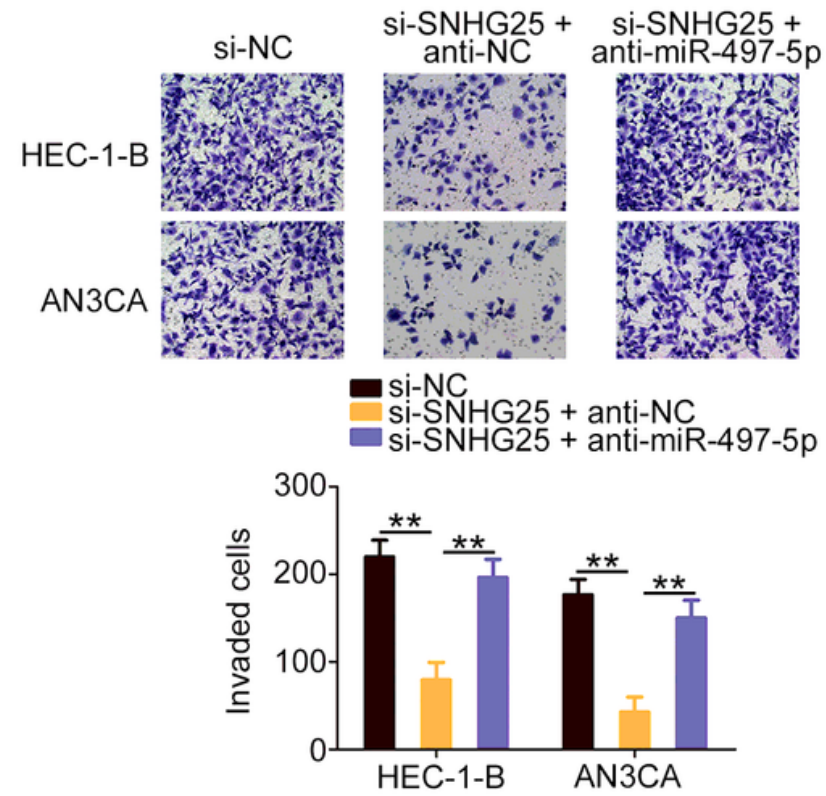

\section{Figure 6}

Inhibition of miR-497-5p abrogated the effects of si-SNHG25 in EC cells. (A) The efficiency of anti-miR497-5p transfection was examined applying qRT-PCR. (B, C) EC cells were transfected with si-NC, siSNHG25+anit-NC, or si-SNHG25+ anti-miR-497-5p, and subjected to the assessment of cell proliferation 
and apoptosis. (D, E) The migratory and invasive abilities were explored in EC cells expressing si-NC, siSNHG25+anit-NC, or si-SNHG25+ anti-miR-497-5p. *P $<0.05$ and ** $P<0.01$.

A

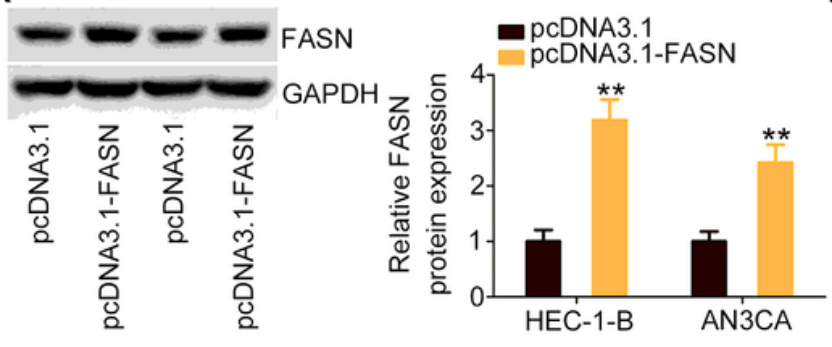

B

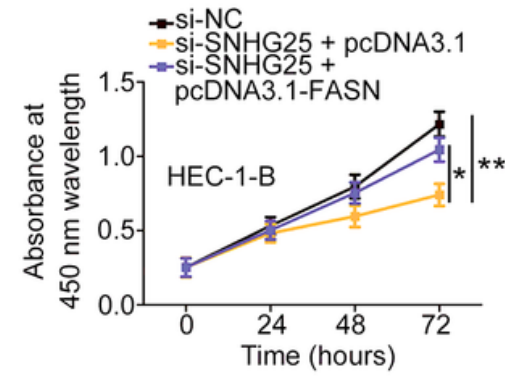

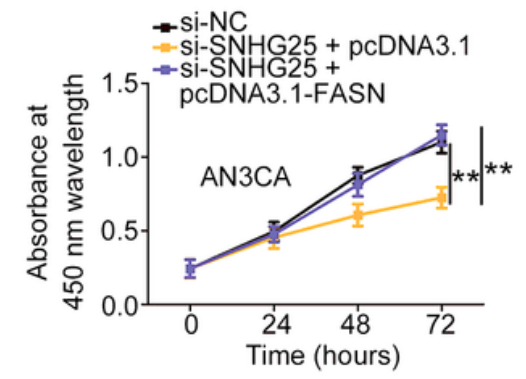

C
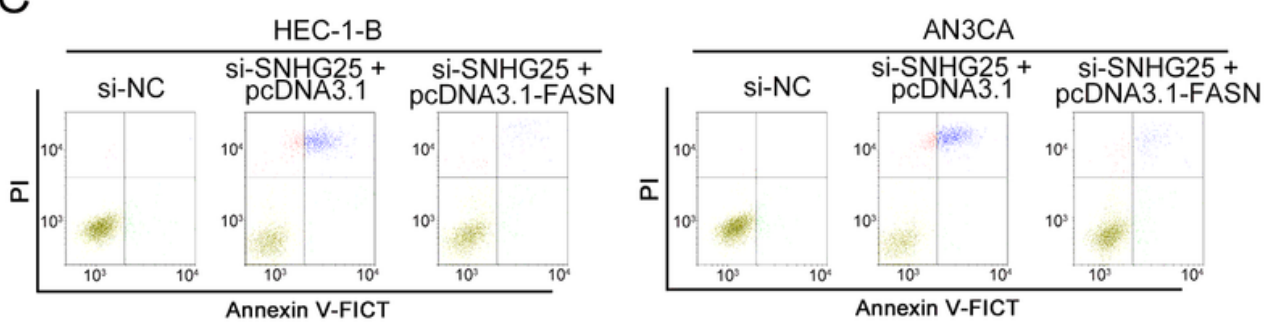

$\mathrm{E}$

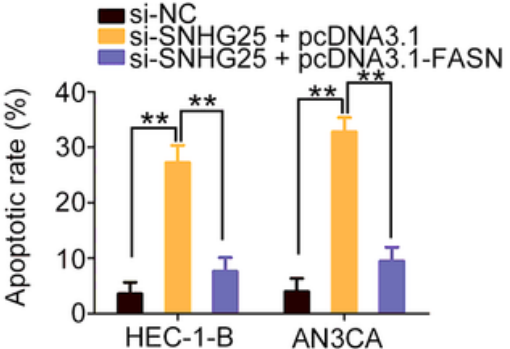

HEC-1-B AN3CA
$\mathrm{D}$

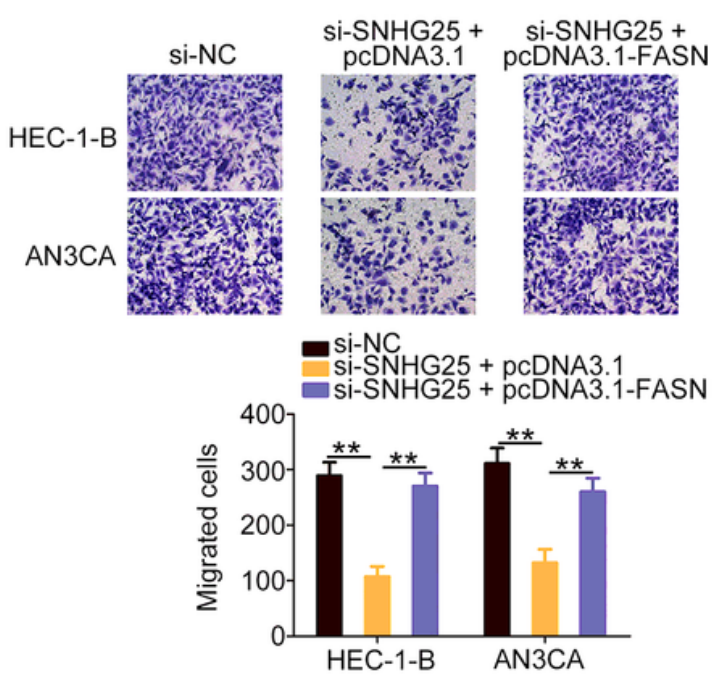

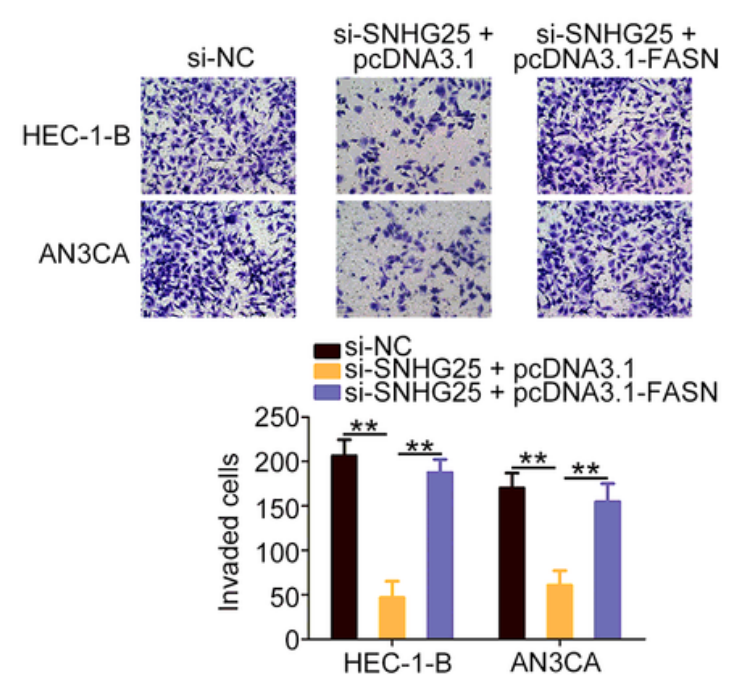

Figure 7

The repressing influences of si-SNHG25 in EC cells were neutralized by FASN restoration. (A) Western blotting was conducted to validate the FASN level in pcDNA3.1-FASN-transfected EC cells. (B-E) EC cells were transfected with si-NC, si-SNHG25+pcDNA3.1, or si-SNHG25+ pcDNA3.1-FASN, followed by the assessment of cell proliferation, apoptosis and motility. ${ }^{\star} P<0.05$ and ${ }^{\star} \mathrm{P}<0.01$. 
A
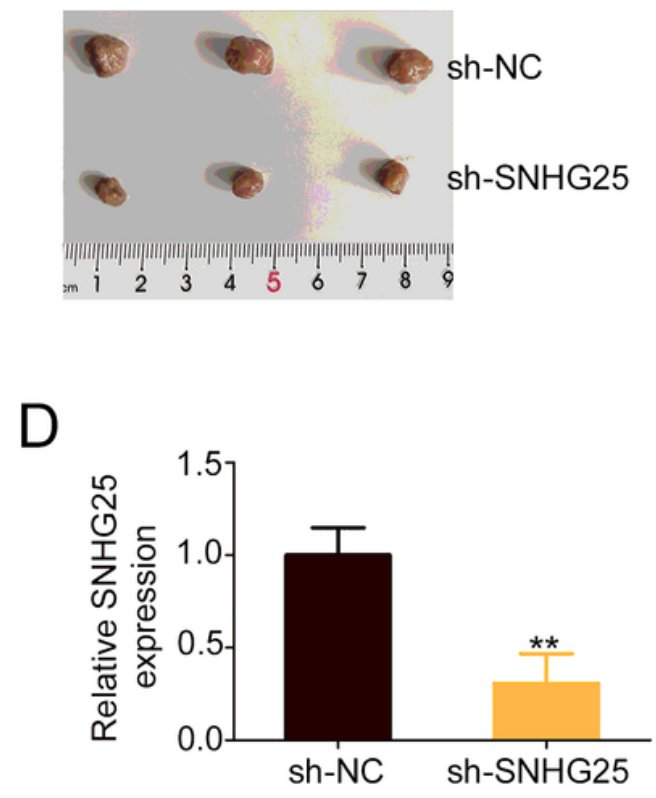

$\mathrm{F}$

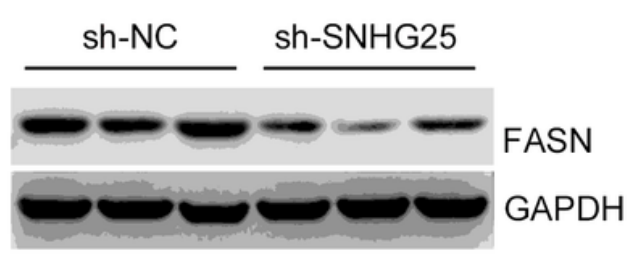

$\mathrm{B}$
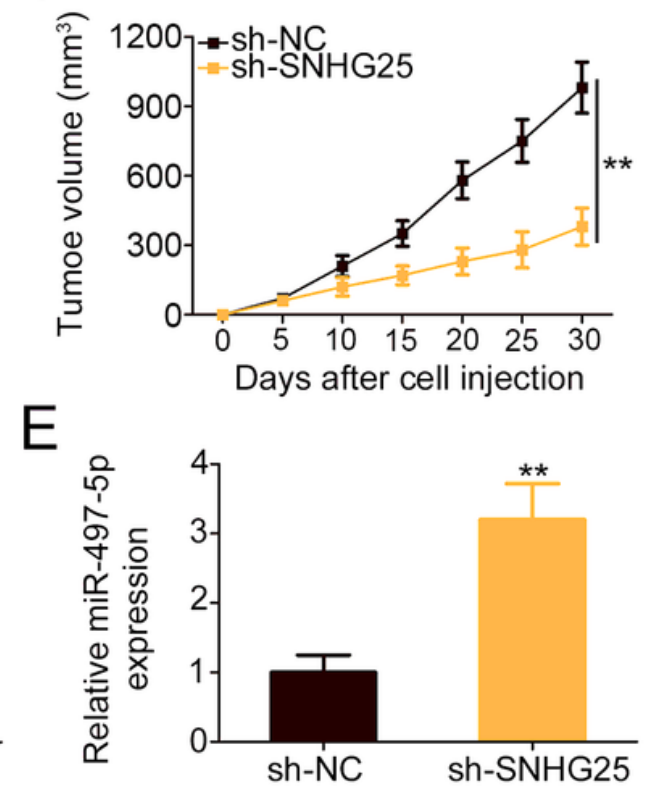

C

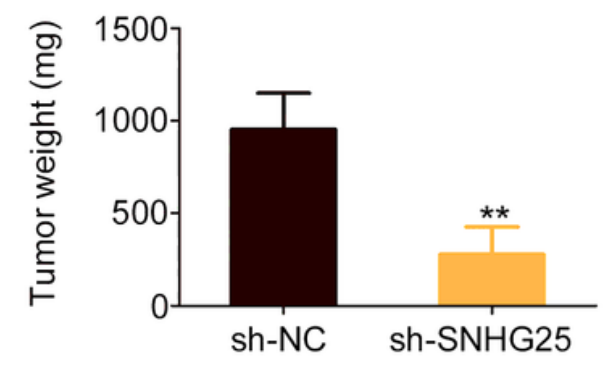

\section{Figure 8}

Downregulation of SNHG25 restrained tumor growth in vivo. (A) Representative images of tumor xenografts resected from mice. (B) Tumor size was recorded every five days, and the data was applied for constructing growth curves. (C) The weight of tumor xenografts was assessed 30 days after treatment. (D, E) Expression of SNHG25 and miR-497-5p in tumor xenografts. ( $F$ ) The protein level of FASN in tumor xenografts. ${ }^{* *} \mathrm{P}<0.01$. 